\title{
Nanoparticle-induced resonant tunneling behaviors in small molecule organic light-emitting devices
}

\author{
Tianhang Zheng, Wallace C. H. Choy, ${ }^{\text {a) }}$ and Yuxiu Sun \\ Department of Electrical and Electronic Engineering, The University of Hong Kong, Pokfulam Road, \\ Hong Kong
}

(Received 5 October 2008; accepted 19 February 2009; published online 25 March 2009)

\begin{abstract}
We report a hybrid nanoparticle/organic device with strong resonant tunneling behavior by introducing ligand-capped $\mathrm{Ag}$ nanoparticles between indium tin oxide and hole transport layer in small molecule organic light-emitting devices. The dependences of resonant tunneling current on the thickness of the organic layers are investigated. For the optimized device, a peak-to-valley current ratio as high as 4.5 and narrow peak width about $1.8 \mathrm{~V}$ are obtained. Combining with the analysis of current-voltage behavior, the mechanism based on the charge trapping effect of Ag nanoparticles is proposed to interpret the operation of the hybrid device. Finally, the emission characteristics of hybrid devices are also studied. (C) 2009 American Institute of Physics. [DOI: 10.1063/1.3099038]
\end{abstract}

Metallic nanoparticles (MNPs) have long been a scientific interest because they exhibit size-dependent electronic, optical, and chemical properties that can be further modified by ligand passivation of nanoparticle surfaces, which are attractive for applications ranging from electronic and optoelectronic devices, photonic structures, molecular catalysts, to chemical sensing. ${ }^{1-4}$ Recently, there are lots of studies on MNPs-organic semiconductor system to fabricate organic memory devices based on organic/MNPs/organic (OMO) structures due to their simplicity and high performance. ${ }^{3-6}$ In their devices, an unusual property of negative differential resistance (NDR) was observed in high conductivity state resulting from charge trapping function of MNPs. Several other groups also demonstrated NDR effect in their organic semiconductor devices by employing superlattice structure, ${ }^{7}$ double barrier structure, ${ }^{8}$ or polymer-MNPs composites. ${ }^{9}$

The NDR effect is an essential characteristic of resonant tunneling devices (RTDs). Such device has promising applications in memory cells, analog oscillators, and functional RTD-based circuits. ${ }^{10}$ However, for the current-voltage characteristics in NDR region of all above devices, the peak-tovalley current ratio (PVCR) of current resonance peak was small at room temperature (generally, lower than 2) and the broadening effect of their spectral linewidth was serious.

In this letter, a hybrid device with the strong resonant tunneling behaviors and a simple structure is demonstrated by introducing silver nanoparticles [Ag nanoparticles (NPs)] between the indium tin oxide (ITO) anode and small molecule organic layered structures rather than using OMO structures. The performance of such organic RTD is optimized, and a high PVCR of more than 4.5 and a narrow linewidth $(\Delta V \approx 1.8 \mathrm{~V})$ of resonant current peak at half height between peak and valley current have been achieved successfully.

The synthesis procedures of Ag NPs referred to the work of Sun et al. ${ }^{11}$ In this process, the surface of Ag NPs would be capped by the organic capping layer. Afterwards, Ag NPs solution was dispersed into ethanol with the $\mathrm{Ag}$ concentration of $1.25 \times 10^{-2} \mathrm{~mol} / \mathrm{l}$. The resulting Ag NPs have a uni-

\footnotetext{
${ }^{\text {a) }}$ Author to whom correspondence should be addressed. Electronic mail: chchoy@eee.hku.hk.
}

form size distribution and no aggregation effect appearing, and the diameter was in the range of $4.5 \pm 0.5 \mathrm{~nm}$, as shown in the transmission electron microscopy image in Fig. 1(a). It can also be verified from the absorption spectrum in the inset of Fig. 1(a), where a typical strong plasmon resonance peak located around $415 \mathrm{~nm}$ with a narrow full width at half maximum was presented.

All devices were fabricated onto $120 \mathrm{~nm}$ ITO coated glass substrates with a sheet resistance of $15 \Omega / \square$. An optimized layer of $\sim 10 \mathrm{~nm} \mathrm{Ag} \mathrm{NPs} \mathrm{was} \mathrm{spin-coated} \mathrm{onto} \mathrm{the}$ cleaned substrates and dried naturally for $30 \mathrm{~min}$ in a glovebox. Organic materials including $N, N^{\prime}$-diphenyl- $N, N^{\prime}$ bis(1-naphthyl)-(1,1'-biphenyl)-4, 4' -diamine (NPB) and tris(8-hydroxyquinoline) aluminum $\left(\mathrm{Alq}_{3}\right)$ were grown sequentially by thermal evaporation. $\mathrm{A} \operatorname{LiF}(1 \mathrm{~nm}) / \operatorname{Al}(100 \mathrm{~nm})$ cathode was deposited and patterned using a mask to define

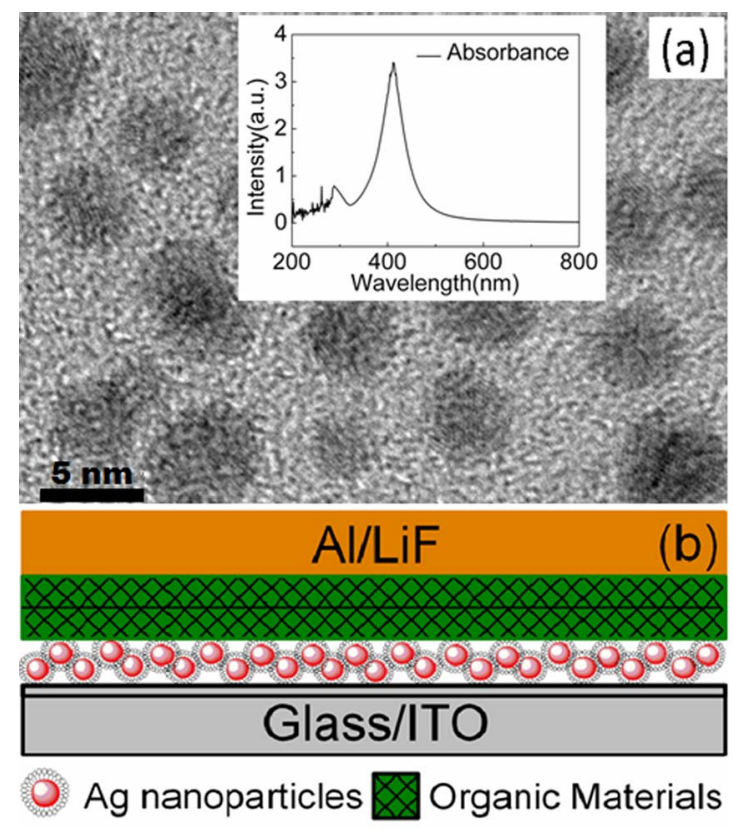

FIG. 1. (Color online) (a) The transmission electron microscope image of $\mathrm{Ag}$ nanoparticles, and the scale bar is $5 \mathrm{~nm}$. Inset: the absorption spectrum of $\mathrm{Ag}$ nanoparticles. (b) The schematic illustration of hybrid device structure. 

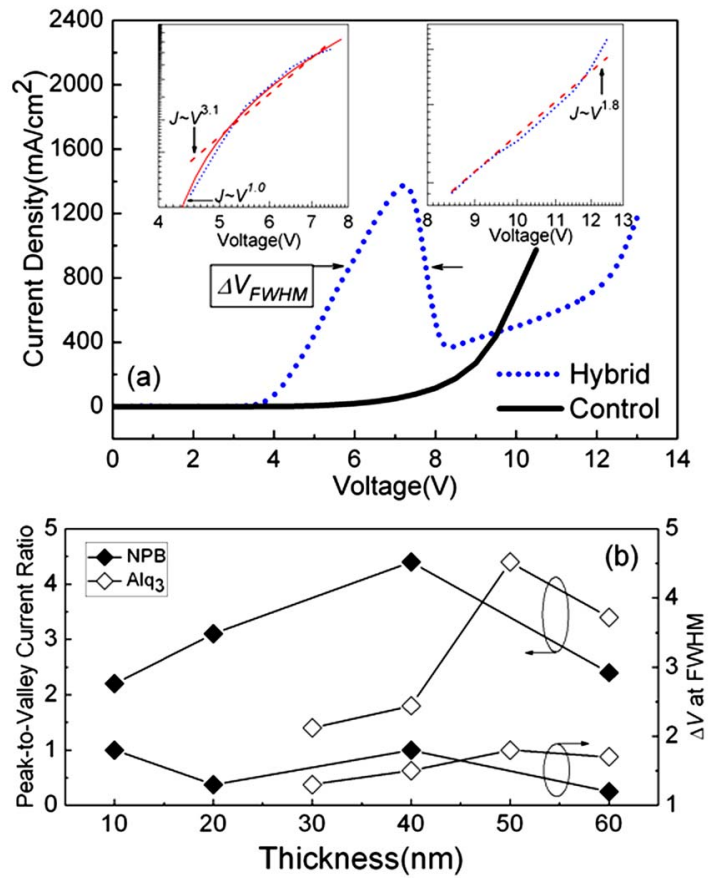

FIG. 2. (Color online) (a) The $J-V$ characteristics of the hybrid and the control devices. The inset shows the fitting results by power law and liner models for $J-V$ in the range of 4.5-7.5 $\mathrm{V}$ (left) and 8.5-12.5 $\mathrm{V}$ (right), respectively. (b) The dependence of PVCR and $\Delta V$ on the thickness of two organic layers.

an active area of $3.57 \mathrm{~cm}^{2}$. These devices have a hybrid structure of $\mathrm{ITO} / \mathrm{AgNPs} / \mathrm{NPB} / \mathrm{Alq}_{3} / \mathrm{LiF} / \mathrm{Al}$, as shown in Fig. 1(b). A control device of $\mathrm{ITO} / \mathrm{NPB}(40 \mathrm{~nm}) /$ $\mathrm{Alq}_{3}(50 \mathrm{~nm}) / \mathrm{LiF}(1 \mathrm{~nm}) / \mathrm{Al}(100 \mathrm{~nm})$ was fabricated. The characteristics of current-voltage-luminance $(J-V-L)$ and electroluminance (EL) spectra were measured by a Keithley 2400 source, a calibrated Si photodiode, and an Oriel spectrometer, respectively. The details can be found elsewhere. ${ }^{12}$ The capacitance-voltage $(C-V)$ tests were carried out using HP4284A precision $L C R$ meter at $10^{6} \mathrm{~Hz}$.

Figure 2(a) shows the $J$ - $V$ characteristics of the optimized hybrid device and the control device where the thickness of NPB and $\mathrm{Alq}_{3}$ is 40 and $50 \mathrm{~nm}$, respectively. A strong current resonant peak appears in 4-8 V range when Ag NPs are introduced between ITO and HTL layer, which is a typical $J$ - $V$ characteristics of an organic RTD. ${ }^{7}$ A PVCR value as high as $\sim 4.5$ and a narrow peak width of around $1.4-1.8 \mathrm{~V}$ have been achieved. ${ }^{13}$ PVCR is an important figure of merit to justify RTD performance that can be used to estimate the maximum oscillation frequency $\left(f_{\max }\right)$ of this device by ${ }^{14}$

$$
f_{\max }=C^{-1} \times \frac{\Delta I}{\Delta V} .
$$

Here, $C$ is the geometric capacitance at the voltage where the maximum negative conductance is obtained. $\Delta I$ and $\Delta V$ are the change in current and voltage in the NDC region, respectively. $C$ is about $700-800 \mathrm{pF}$ at the maximum negative conductance. Hence, the $f_{\max }$ of $0.064-0.11 \mathrm{GHz}$ can be expected from this hybrid device at room temperature.

Such high performance is achieved by optimizing the thickness of two organic layers, respectively. We find that PVCR is more dependent on the thicknesses of NPB and $\mathrm{Alq}_{3}$ as compared with the variation in $\Delta V$, as shown in Fig. 2(b). For the device with $50 \mathrm{~nm} \mathrm{Alq}$, when NPB changes

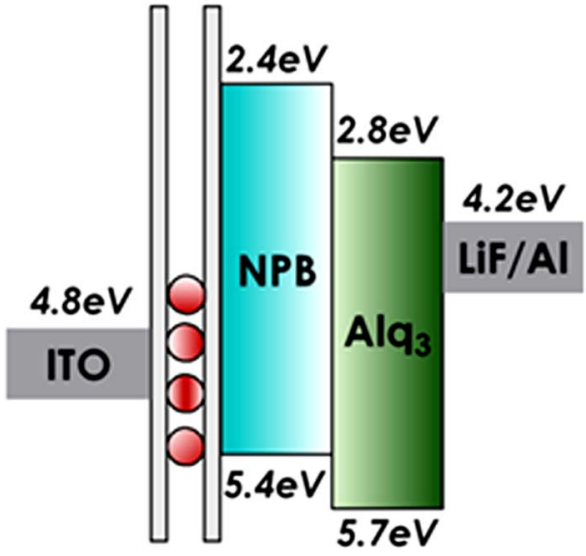

FIG. 3. (Color online) Energy level diagram of the hybrid device.

from 10, 20, and 40 to $60 \mathrm{~nm}$, PVCR increases first to its maximum at $40 \mathrm{~nm}$ and then starts to decrease. The same trend has been observed when $\mathrm{Alq}_{3}$ increases from 30, 40, and 50 to $60 \mathrm{~nm}$ for the device with $40 \mathrm{~nm}$ NPB. Thus, the optimized point is obtained where NPB and $\mathrm{Alq}_{3}$ is 40 and $50 \mathrm{~nm}$, respectively.

Compared with the $J-V$ characteristic of the control device, the hybrid device exhibits several distinct features. First, it has a higher current density in low bias range from 4 to $8 \mathrm{~V}$, and the current is nearly proportional to the applied bias and follow the $J \propto V^{1.0}$ relation precisely, as shown in the inset of Fig. 2(a). This demonstrates that the device shows a near linear current which is different from typical behavior of a semiconductor device. ${ }^{15}$ A power law fit has also been carried out giving $J \propto V^{3.1}$ (see the inset of Fig. 1), but it still deviates from experiment results seriously, which demonstrates that the current behavior is different from field dependent space charge limited current model (SCLC). New resonant current channel should be formed after the introduction of Ag NPs layer. The details will be discussed below. Second, between 7 and $8 \mathrm{~V}$, it is in the NDR region where the current decreases rapidly to its valley value. Third, with the further increasing of bias, the current also increases slowly. When we fit this region using power law relationship, $J \infty V^{1.8}$ has been obtained, which approaches a square law $\left(J \infty V^{2.0}\right)$ illustrating the current in this region follows a SCLC model like an organic light-emitting diode (OLED). ${ }^{16}$

The unusual properties in hybrid devices can be interpreted by the mechanism as proposed below. From various studies about OMO structures, ${ }^{3-6}$ we know that MNPs can function as deep charge trapping sites to store a great deal of carriers stably, especially with a capping layer. This effect can also happen in our case, although we introduce Ag NPs between ITO and HTL. Figure 3 shows the schematic energy diagram of the hybrid device. There, Ag NPs form several discrete trapping levels, and the trapping level can be aligned with the highest occupied molecular orbital of NPB when the value of voltage is satisfied. Thus, the resonant channel is formed and the current reaches to the peak point following a near linear relation like a capacitor behavior. Above this voltage, the energy in resonant channel falls into the trapping state of Ag NPs that will raise the interfacial barrier to make the current decrease, and thus NDR occurs. It is noteworthy that the stored carriers in Ag NPs should be holes rather than electrons, and the later will accumulate in the $\mathrm{Alq}_{3}$ layer and cannot pass through this layer due to the effective electron 

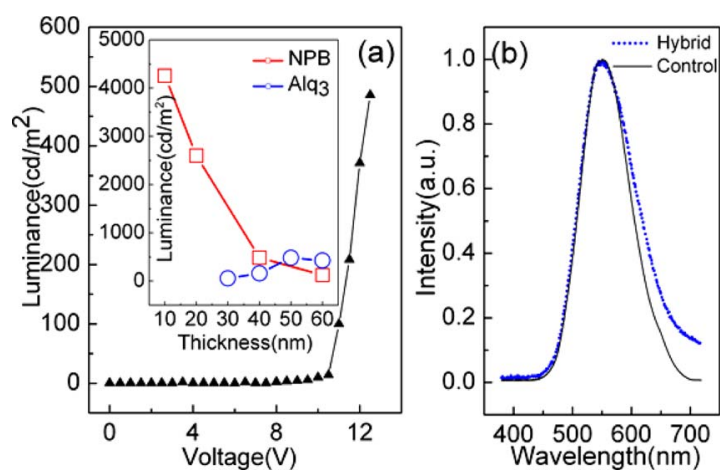

FIG. 4. (Color online) (a) The $L-V$ characteristic of the hybrid device. Inset: the dependence of luminance on the thicknesses of NPB and $\mathrm{Alq}_{3}$, respectively. The thickness of NPB is changed from 10 to $60 \mathrm{~nm}$ when $\mathrm{Alq}_{3}$ is fixed at $50 \mathrm{~nm}$. Besides, the thickness of $\mathrm{Alq}_{3}$ is adjusted from 30 to $60 \mathrm{~nm}$ when NPB is fixed at $40 \mathrm{~nm}$. (b) EL spectra of hybrid and control devices.

blocking effect (energy barrier around $0.4 \mathrm{eV}$ ) of NPB layer in $\mathrm{NPB} / \mathrm{Alq}_{3}$ interface. ${ }^{3,17}$ Consequently, there is no light emission because holes and electrons cannot efficiently recombine to form excitons. With the bias increasing further, the stored holes are driven out of Ag NPs and begin to transport through NPB layer toward cathode side and recombine with the accumulated electrons to form excitons at the interface of $\mathrm{NPB} / \mathrm{Alq}_{3}$ to emit light eventually, which results in a square law relation of $J-V$ in this region. In addition, for the reason of the dependence of PVCR on the thickness of two organic layers, we know that the carrier transport, interface charging, and blocking effects can be influenced by different thicknesses of organic layers, either NPB or $\mathrm{Alq}_{3}$. Thin NPB layer not only cannot block electrons effectively, which leads to electron leaking into NPB and recombined with the trapped holes that existed in the Ag NPs, but also it will enhance interfacial scattering effects. ${ }^{17,18}$ When $\mathrm{Alq}_{3}$ layer is thin, similar issues of interfacial scattering and electron penetration into NPB also exist. All of these reasons result in a small PVCR. However, for thick NPB or $\mathrm{Alq}_{3}$ layer, the field strength becomes weak at the same bias, which cannot provide enough momentum energy for charge tunneling and thus results in small PVCR. The resonant current peak position also shifts toward high voltage in 1.5-2.0 V range with thickness increasing, even those results have not been shown here. Further studies are in progress to clarify the concrete mechanism of this dependence. A near constant $\Delta V$ demonstrates that the trapped energy of Ag NPs for these devices is equivalent basically according to the relation of $\Delta V=\Delta E / e$, where $\Delta E$ and $e$ stand for the energy difference of different states and electron, respectively. ${ }^{19}$

Figure 4 shows the $V-L$ characteristic and EL spectra of the hybrid device. There is no light emission in the resonant peak region, suggesting that the resonant tunneling current cannot contribute to the luminance due to the absence of hole carriers by the strong trapping effect of Ag NPs. When the bias is further increased, the carrier recombination increases and a maximum luminance at around $500 \mathrm{~cd} / \mathrm{m}^{2}$ has been obtained in green color. The slight difference of EL spectra between hybrid and control devices may result from structure deviation and scattering of Ag NPs. The dependence of luminance on the thickness of NPB and $\mathrm{Alq}_{3}$ is shown in the inset of Fig. 4(a). The thickness of NPB is changed from 10 to $60 \mathrm{~nm}$ when $\mathrm{Alq}_{3}$ is $50 \mathrm{~nm}$. Besides, the thickness of $\mathrm{Alq}_{3}$ is adjusted from 30 to $60 \mathrm{~nm}$ when NPB layer is $40 \mathrm{~nm}$. We can see the luminance decreases with increasing NPB thickness. This illustrates that hole is the minority compared with electron, and partial holes still reside in Ag NPs after the resonant tunneling occurs. This speculation has been confirmed in another work. Therefore, higher luminance can be achieved for devices with thick $\mathrm{Alq}_{3}$ layer. Further enhancement can be expected by balancing hole and electron species better or doping high efficient emitters, while resonant tunneling behavior is still available.

In conclusion, we demonstrated a robust hybrid nanoparticle/organic device with strong resonant tunneling behavior. The PVCR and the emission properties of hybrid devices are intensively dependent on the variations in the thickness of two organic layers, and an optimized point is obtained where the device has a PVCR as high as 4.5 and narrow peak width $(\Delta V \approx 1.8 \mathrm{~V})$ of resonant tunneling current, which shows promise as a RTD for practical applications. With the analysis of current-voltage behavior, the mechanism based on the charge trapping effect of Ag NPs make the hybrid device exhibit the dual features like a capacitor to trap carriers and an OLED to emit light in low and high bias regions, respectively. To our knowledge, this is the first demonstration of an organic RTD with simple structure and high performance at room temperature.

This work is supported by Grant No. 14300.324.01 from the Research Grant Council of the HK Special Administrative Region, China. We would like to thank Professor P. T. Lai for his technical assistance in the $C$ - $V$ measurement.

${ }^{1}$ S. Chen, R. W. Murray, and S. W. Feldberg, J. Phys. Chem. B 102, 9898 (1998).

${ }^{2}$ S. Kuhn, U. Hakanson, L. Rogobete, and V. Sandoghdar, Phys. Rev. Lett. 97, 017402 (2006).

${ }^{3}$ R. J. Tseng, J. Ouyang, C. W. Chu, J. Huang, and Y. Yang, Appl. Phys. Lett. 88, 123506 (2006).

${ }^{4}$ C. H. Tu, D. L. Kwong, and Y. S. Lai, Appl. Phys. Lett. 89, 252107 (2006).

${ }^{5}$ L. D. Bozano, B. W. Kean, V. R. Deline, J. R. Salem, and J. C. Scott, Appl. Phys. Lett. 84, 607 (2004).

${ }^{6}$ J. Ouyang, C. W. Chu, D. Sieves, and Y. Yang, Appl. Phys. Lett. 86, 123507 (2005).

${ }^{7}$ T. J. Park, Y. K. Lee, S. K. Kwon, J. H. Kwon, and J. Jang, Appl. Phys. Lett. 89, 151114 (2006).

${ }^{8}$ S. Y. Ryu, S. J. Jo, C. S. Kim, S. H. Choi, J. H. Noh, H. K. Baik, H. S. Jeong, D. W. Han, S. Y. Song, and K. S. Lee, Appl. Phys. Lett. 91, 093515 (2007).

${ }^{9}$ T. Kondo, S. M. Lee, M. Malicki, B. Domercq, S. R. Marder, and B. Kippelen, Adv. Funct. Mater. 18, 1112 (2008).

${ }^{10}$ J. P. Sun, G. I. Haddad, P. Mazumder, and J. N. Schulman, Proc. IEEE 86, 641 (1998).

${ }^{11}$ S. Sun, H. Zeng, D. B. Robinson, S. Raoux, P. M. Rice, S. X. Wang, and G. Li, J. Am. Chem. Soc. 126, 273 (2004).

${ }^{12}$ T. H. Zheng and W. C. H. Choy, J. Phys. D 41, 055103 (2008).

${ }^{13}$ W. C. H. Choy and T. H. Zheng, U.S. Provisional Patent Application No. 61/097, 405 (2008).

${ }^{14}$ M. W. Dashiell, J. Kolodzey, P. Crozat, F. Aniel, and J. M. Lourtioz, IEEE Electron Device Lett. 23, 357 (2002).

${ }^{15}$ T. Matsushima, Y. Kinoshita, and H. Murata, Appl. Phys. Lett. 91, 253504 (2007)

${ }^{16}$ H. Yamamoto, H. Kasajima, W. Yokoyama, H. Sasabe, and C. Adachi, Appl. Phys. Lett. 86, 083502 (2005).

${ }^{17}$ S. T. Zhang, Z. J. Wang, J. M. Zhao, Y. Q. Zhan, Y. Wu, Y. C. Zhou, X. M. Ding, and X. Y. Hou, Appl. Phys. Lett. 84, 2916 (2004).

${ }^{18}$ S. C. Tse, H. H. Fong, and S. K. So, J. Appl. Phys. 94, 2033 (2003).

${ }^{19}$ S. Luryi, Appl. Phys. Lett. 47, 490 (1985). 